\title{
Wnt Signaling in Neural Circuit Development
}

\author{
Lee G. Fradkin, ${ }^{1}$ Gian Garriga, ${ }^{2}$ Patricia C. Salinas, ${ }^{3}$ John B. Thomas, ${ }^{4}$ Xiang Yu, ${ }^{5}$ and Yimin Zou ${ }^{6}$ \\ ${ }^{1}$ Department of Molecular and Cell Biology, Leiden University Medical Center, 2333 AL, Leiden, The Netherlands, ${ }^{2}$ Department of Molecular and Cell \\ Biology, University of California, Berkeley, Berkeley, California 94720, ${ }^{3}$ Department of Anatomy and Developmental Biology, University College London, \\ London WC1E 6BT, United Kingdom, ${ }^{4}$ Salk Institute, San Diego, California 92186, ${ }^{5}$ Institute of Neuroscience, Chinese Academy of Sciences, Shanghai \\ 200031, China, and ' Department of Neurobiology, Pharmacology, and Physiology, The University of Chicago, Chicago, Illinois 60637
}

Key words: neuronal migration; axon guidance; dendrite morphogenesis; synapse formation; Derailed; Frizzled; Ryk; Wnt; Disheveled

The Wingless-type (Wnt) family proteins are well known for their functions in embryonic patterning, cell fate determination, and cell proliferation (Wodarz and Nusse, 1998). Wnts act through both canonical and noncanonical signal transduction pathways. Canonical Wnt signaling results in the stabilization of $\beta$-catenin, which translocates to the nucleus and forms a complex with the ternary complex factor-lymphoid enhancer factor transcription factor to differentially regulate target genes, whereas the noncanonical pathways are mediated through Rac/Rho or through calcium.

Recent studies in a number of systems led to the surprising finding that Wnts play important roles in several aspects of neural circuit development, such as neuronal migration, axon pathfinding, dendritic morphogenesis, and synaptic differentiation (Schnorrer and Dickson, 2004; Yoshikawa and Thomas, 2004; Zou, 2004; Ciani and Salinas, 2005; Charron and Tessier-Lavigne, 2005). This type of molecular pleiotropy (producing more than one effect) is a common theme and has been observed in other molecular families, such as FGFs, bone morphogenetic proteins, and neurotrophins.

Studying the function and mechanisms of Wnts in these later processes of nervous system development is shedding new light on how intricate neuronal networks are assembled and how specific downstream signaling pathways mediate these diverse functions. Importantly, elucidating the molecular and cellular mechanisms of Wnt action during nervous system development could provide new insights to the functional organization of the nervous system and allow the design of therapeutic strategies for neural circuit repair after injury or disease. The purpose of this mini-symposium was to bring together researchers in this dynamic area to discuss their latest findings, consolidate hypotheses, promote collaborations, and expedite research progress. This mini-symposium summarizes the emerging themes represented and then discusses future directions.

\section{Conservation}

A prominent theme is that much of the function and signaling mechanism of Wnt proteins in these processes appears highly conserved. This is particularly evident in axon guidance; studies

Received Aug. 15, 2005; revised Sept. 13, 2005; accepted Sept. 17, 2005

Correspondence should be addressed to Yimin Zou, Department of Neurobiology, Pharmacology, and Physiology, The University of Chicago, 924 East 57th Street, R216, Chicago, IL 60637. E-mail: yzou@bsd.uchicago.edu. DOI:10.1523/JNEUROSCI.3429-05.2005

Copyright $\odot 2005$ Society for Neuroscience $\quad$ 0270-6474/05/2510376-03\$15.00/0 in both invertebrates and vertebrates demonstrated that Wnts are axon guidance molecules. In Drosophila embryos, commissural axons cross the midline through either the anterior or the posterior commissure in each segment of the ventral nerve cord. Derailed (Drl), a member of the conserved Ryk receptor tyrosine kinase-related (Ryk) "fractured" family of proteins, controls commissure selection. Drl-positive neurons normally cross the midline through the anterior commissure (Callahan et al., 1995; Bonkowsky et al., 1999). In a screen for genes interacting with derailed, Wnt5 was found to encode a Drl ligand that regulates midline crossing by preventing Drl-positive commissural axons from crossing the midline through the posterior commissure (Yoshikawa et al., 2003). The expression of Wnt5 in the posterior commissural led to the postulation that the anterior commissural selection is mediated by Wnt5 repulsive function through Derailed (Yoshikawa et al., 2003). A Wnt5-mediated defasciculation function has also been proposed that may contribute to the midline pathway selection (Fradkin et al., 2004). Wnt5 expression itself is transcriptionally repressed by Derailed in anterior commissural neurons (Fradkin et al., 2004). The existence of such a regulatory mechanism suggests that Wnt5 expression may be required for other aspects of anterior commissure development.

In vertebrates, Wnts were found to direct the anterior turning of spinal cord commissural axons after midline crossing (Lyuksyutova et al., 2003). Axonal connections are organized along the anteroposterior and dorsoventral axes in the CNS. Spinal cord commissural axons first project along the dorsoventral axis, reach the ventral midline, and then turn anteriorly after midline crossing. Postcrossing axons become attracted by several Wnts, particularly Wnt4 and Wnt7b, which are expressed in anteroposterior decreasing gradients along the ventral midline. This attraction is mediated by a Wnt receptor, Frizzled 3. More recently, corticospinal tract axons, which project in the opposite direction along the length of the spinal cord, were also found to be guided by Wnts. These axons are repelled by Wnt 1 and Wnt5a, which are expressed in an anteroposterior decreasing gradient and control the posterior-directed growth along the dorsal midline. This Wnt repulsion is mediated by Ryk, the mammalian homolog of Derailed (Liu et al., 2005).

In Caenorhabditis elegans, Wnt-Frizzled pathways appear to be involved in axon projection along the anteroposterior body axis as well. Several Wnts and Frizzleds control the anterior growth of ALM, AVM, and PVM axons. Because of redundancy, loss of more than one Wnt or Frizzled often leads to premature 
termination and rerouting phenotypes. Additionally, ectopic expression of endoglycanase-20 (EGL-20) (C. elegans Wnt) indicates that it can act as a repellent for AVM and PVM growth cones (G. Garriga, unpublished results).

\section{Versatility}

Wnts are highly versatile, suggesting the importance of this family of signaling molecules in nervous system development. Wnts are not only required for axon guidance but are also involved in neuronal migration, synaptic differentiation, and dendritic morphogenesis.

Cell migration controls neuronal positioning, a critical step in circuit development. In C. elegans, EGL-20/Wnt controls the migration of hermaphrodite-specific (HSN) neurons and Q neuroblasts along the anteroposterior axis (Desai et al., 1988; Harris et al., 1996; Maloof et al., 1999). The posterior migration of QL by EGL-20/Wnt is mediated by the canonical Wnt signaling pathway, which transcriptionally regulates genes necessary for posterior migration. Although the signaling mechanism of anterior migration of HSN and QR is currently not known, Wntmediated repulsion appears to guide the HSNs anteriorly.

Once specific contacts have been established, synaptic assembly takes place. This process requires the dialog between the presynaptic and postsynaptic targets. Previous studies showed that Wnt7a, secreted by postsynaptic granule cells in cerebellum, acts as a synaptogenic factor to regulate morphological remodeling of presynaptic mossy fibers (Hall et al., 2000). New gain-of-function and loss-of-function studies in the mouse show that Wnt7a through Disheveled ( $\mathrm{Dvl}$ ) regulates presynaptic morphology and the assembly of the presynaptic apparatus in the mouse cerebellum (P. C. Salinas, unpublished results). In the spinal cord, Wnt3 was also found to function as a retrograde signal that regulates terminal arborization of neurotrophin-3-responsive spinal sensory neurons before the formation of sensory motoneuron synapses (Krylova et al., 2002). At the Drosophila neuromuscular junction, loss of Wingless, which is normally secreted by presynaptic boutons, results in dramatic reductions in target-dependent synapse formation and loss or aberrant development of active zones and postsynaptic specializations (Packard et al., 2003). These findings demonstrate that Wnts can function at the central and peripheral synapses as retrograde or anterograde signals.

Wnt signaling also regulates dendrite morphogenesis. In cultured hippocampal neurons, dendritic morphogenesis requires the function of $\beta$-catenin through a transcription-independent mechanism, which likely involves the cadherins/catenin cell adhesion complexes (Yu and Malenka, 2003). Interestingly, Wnt signaling through Disheveled, Rac, and c-Jun N-terminal protein kinase (JNK) also regulates dendritic development in cultured hippocampal neurons. Importantly, deficiency in Disheveled-1 results in poor dendritic development (Rosso et al., 2005). Additional studies will be needed to address whether and how these two pathways regulate different aspects of dendritic development.

One explanation for the diversity of functions could be that there are multiple members of both the ligand and receptor families, which are expressed in many areas during various developmental stages, even in adulthood. In mammals, there are 19 Wnts, 10 Frizzleds, and one Ryk/Derailed. In fly, there are seven Wnts, four Frizzleds, and two Ryks. In C. elegans, there are five Wnts, four Frizzles, and one Ryk.

\section{Specificity}

The multiple Wnt functions in circuit development are likely mediated by distinct and specific signaling pathways. A major challenge is to sort out the signaling mechanisms that account for the diverse functions of Wnts. Two major classes of receptors mediate Wnt action, Frizzleds and Ryk/Derailed. Both classes of receptors are also involved in earlier functions, such as cell fate determination (Wodarz and Nusse, 1998; Halford et al., 2000; Inoue et al., 2004). There is no compelling evidence that Drosophila frizzled genes interact with derailed (Yoshikawa et al., 2003). However, the mammalian Ryk protein has been demonstrated to form a ternary complex in transfected cells with Frizzled and Wnt, but the functional consequences of Ryk-Frizzled interaction were not reported ( $\mathrm{Lu}$ et al., 2004). Knowing the downstream signaling mechanisms of Ryk will be key to understanding Wnt functions in CNS development.

Studies are already underway to address these questions. In growth cone guidance, Frizzled is required for attraction whereas Derailed is required for repulsion, and these two receptors likely have their own downstream effectors (Zou, 2004). It is currently not known whether these receptors interact with each other in axon guidance or how signaling is integrated in the growth cone. In a screen using an overexpression system of Wnt5 in midline glia cells, which leads to a failure in anterior commissure formation, the membrane bound $\mathrm{O}$-acyl transferase, porcupine, was found to be required for Wnt5 signaling in axon guidance (Fradkin et al., 2004).

In rodents, dendrite morphogenesis is regulated through two signaling pathways, one acting through $\beta$-catenin/cadherin and the other through Dvl-Rac-JNK. Interestingly, the canonical Wnt signaling pathway through transcriptional regulation is not involved in dendritic branching (Yu and Malenka, 2003; Rosso et al., 2005). Identification of divergent points for signaling pathways will be essential for understanding the specificity of Wnt signaling. How canonical Wnt signaling leads to directional neuronal migration in C. elegans is another fascinating question. The study of the signaling mechanisms for almost all Wnt-mediated processes is in its infancy.

In closing, the finding that Wnts are involved in later development may be after all inevitable in light of the complexity of the nervous system and limited number of genes in any given genome. It is our consensus that we are only scratching the surface in understanding the roles and mechanisms of Wnt signaling in neural circuit development and function. Additional studies of these questions will enhance our knowledge of the nervous system structure and function.

\section{References}

Bonkowsky JL, Yoshikawa S, O’Keefe DD, Scully AL, Thomas JB (1999) Axon routing across the midline controlled by the Drosophila Derailed receptor. Nature 402:540-544.

Callahan CA, Muralidhar MG, Lundgren SE, Scully AL, Thomas JB (1995) Control of neuronal pathway selection by a Drosophila receptor proteintyrosine kinase family member. Nature 376:171-174.

Charron F, Tessier-Lavigne M (2005) Novel brain wiring functions for classical morphogens: a role as graded positional cues in axon guidance. Development 132:2251-2262.

Ciani L, Salinas PC (2005) WNTs in the vertebrate nervous system: from patterning to neuronal connectivity. Nat Rev Neurosci 6:351-362.

Desai C, Garriga G, McIntire SL, Horvitz HR (1988) A genetic pathway for the development of the Caenorhabditis elegans HSN motor neurons. Nature 336:638-646.

Fradkin LG, van Schie M, Wouda RR, de Jong A, Kamphorst JT, RadjkoemarBansraj M, Noordermeer JN (2004) The Drosophila Wnt5 protein mediates selective axon fasciculation in the embryonic central nervous system. Dev Biol 272:362-375.

Halford MM, Armes J, Buchert M, Meskenaite V, Grail D, Hibbs ML, Wilks AF, Farlie PG, Newgreen DF, Hovens CM, Stacker SA (2000) Ryk- 
deficient mice exhibit craniofacial defects associated with perturbed Eph receptor crosstalk. Nat Genet 25:414-418.

Hall AC, Lucas FR, Salinas PC (2000) Axonal remodeling and synaptic differentiation in the cerebellum is regulated by WNT-7a signaling. Cell 100:525-535.

Harris J, Honigberg L, Robinson N, Kenyon C (1996) Neuronal cell migration in C. elegans: regulation of Hox gene expression and cell position. Development 122:3117-3131.

Inoue T, Oz HS, Wiland D, Gharib S, Deshpande R, Hill RJ, Katz WS, Sternberg PW (2004) C. elegans LIN-18 is a Ryk ortholog and functions in parallel to LIN-17/Frizzled in Wnt signaling. Cell 118:795-806.

Krylova O, Herreros J, Cleverley KE, Ehler E, Henriquez JP, Hughes SM, Salinas PC (2002) WNT-3, expressed by motoneurons, regulates terminal arborization of neurotrophin-3-responsive spinal sensory neurons. Neuron 35:1043-1056.

Liu Y, Shi J, Lu C, Wang Z, Lyuksyutova AI, Song X, Zou Y (2005) Rykmeidated Wnt repulsion regulates posterior-directed growth of corticospinal tract in the spinal cord. Nat Neurosci 8:1151-1159.

Lu W, Yamamoto V, Ortega B, Baltimore D (2004) Mammalian Ryk is a Wnt coreceptor required for stimulation of neurite outgrowth. Cell 119:97-108.

Lyuksyutova AI, Lu CC, Milanesio N, King LA, Guo N, Wang Y, Nathans J,
Tessier-Lavigne M, Zou Y (2003) Anterior-posterior guidance of commissural axons by Wnt-frizzled signaling. Science 302:1984-1988.

Maloof JN, Whangbo J, Harris JM, Jongeward GD, Kenyon C (1999) A Wnt signaling pathway controls hox gene expression and neuroblast migration in C. elegans. Development 126:37-49.

Packard M, Mathew D, Budnik V (2003) Wnts and TGF beta in synaptogenesis: old friends signalling at new places. Nat Rev Neurosci 4:113-120.

Rosso SB, Sussman D, Wynshaw-Boris A, Salinas PC (2005) Wnt signaling through Dishevelled, Rac and JNK regulates dendritic development. Nat Neurosci 8:34-42.

Schnorrer F, Dickson BJ (2004) Axon guidance: morphogens show the way. Curr Biol 14:R19-R21.

Wodarz A, Nusse R (1998) Mechanisms of Wnt signaling in development. Annu Rev Cell Dev Biol 14:59-88.

Yoshikawa S, Thomas JB (2004) Secreted cell signaling molecules in axon guidance. Curr Opin Neurobiol 14:45-50.

Yoshikawa S, McKinnon RD, Kokel M, Thomas JB (2003) Wnt-mediated axon guidance via the Drosophila Derailed receptor. Nature 422:583-588.

Yu X, Malenka RC (2003) Beta-catenin is critical for dendritic morphogenesis. Nat Neurosci 6:1169-1177.

Zou Y (2004) Wnt signaling in axon guidance. Trends Neurosci 27:528532. 